\title{
Delamanid improves outcomes and reduces mortality in multidrug-resistant
} tuberculosis

\author{
Vija Skripconoka*, Manfred Danilovits", Lea Pehme", Tarmo Tomson", \\ Girts Skenders*, Tiina Kummik\#, Andra Cirule*, Vaira Leimane*, Anu Kurve \\ Klavdia Levina", Lawrence J. Geiter ${ }^{+}$, Davide Manissero ${ }^{\S}$ and Charles D. Wells
}

ABSTRACT: Multidrug-resistant and extensively drug-resistant tuberculosis (TB) are associated with worse treatment outcomes for patients, including higher mortality, than for drug-sensitive tuberculosis. Delamanid (OPC-67683) is a novel anti-TB medication with demonstrated activity against multidrug-resistant disease.

Patients who participated in the previously reported randomised, placebo-controlled trial of delamanid and the subsequent open-label extension trial were eligible to participate in a 24-month observational study designed to capture treatment outcomes. Treatment outcomes, as assessed by clinicians and defined by the World Health Organization, were categorised as favourable and unfavourable. Delamanid treatment groups were combined for analysis, based on their duration of treatment. In total, for $421(87.5 \%)$ out of 481 patients from the original randomised controlled trial, consent was granted for follow-up assessments.

Favourable outcomes were observed in 143 (74.5\%) out of 192 patients who received delamanid for $\geqslant 6$ months, compared to $126(55 \%)$ out of 229 patients who received delamanid for $\leqslant 2$ months. Mortality was reduced to $1.0 \%$ among those receiving long-term delamanid versus short-term/no delamanid (8.3\%; $\mathbf{p}<\mathbf{0 . 0 0 1 )}$. Treatment benefit was also seen among patients with extensively drug-resistant TB.

This analysis suggests that treatment with delamanid for 6 months in combination with an optimised background regimen can improve outcomes and reduce mortality among patients with both multidrug-resistant and extensively drug-resistant TB.

KEYWORDS: Extensively drug-resistant, mycobacterium, pulmonary infection, treatment outcomes

M ultidrug-resistant tuberculosis (MDR$\mathrm{TB})$, or tuberculosis (TB) caused by strains of Mycobacterium tuberculosis (MTB) resistant to at least isoniazid and rifampicin, the two most effective bactericidal agents currently available for TB treatment, has emerged as a global public health emergency [1]. It requires treatment with combination therapy consisting of four to six medications including a fluoroquinolone and an injectable anti-TB agent, as well as bacteriostatic agents administered for up to 2 years [2]. Additionally, the treatment is generally more toxic and far more expensive than the standardised treatment regimen used to treat drug-susceptible TB $[3,4]$. Moreover, the inability to use isoniazid and rifampicin for treatment results in a lower likelihood of patients achieving treatment success (bacteriologic cure and treatment completion) and higher mortality than for patients with drugsusceptible TB. In contrast to drug-susceptible TB patients, for whom $\geqslant 85 \%$ can readily achieve treatment success and generally $<5 \%$ die [1], three large meta-analyses of MDR-TB treatment cohorts have shown favourable outcomes in the range of $54 \%$ to $67 \%$ while mortality ranges from $9 \%$ to $15 \%$ [5-7]. Further analyses have shown that if patients fail to achieve sputum culture conversion (SCC) from growth of MTB to no growth of MTB early in the course of MDR-TB treatment, they have a much higher likelihood of a poor outcome at the end of treatment, including death $[8,9]$. Even in high resource settings such as the European Union (EU), surveillance data showed that treatment success averaged from $30-49 \%$ for 2007-2008 MDR-TB cohorts [10,11], although under reporting of treatment outcomes may have affected these results [12].

Extensively drug-resistant (XDR)-TB, or MDR-TB that is also resistant to a fluoroquinolone and an injectable anti-TB agent, has emerged as a more
AFFILIATIONS

${ }^{*}$ Riga East University Hospital, Centre of Tuberculosis and Lung Diseases, Riga, Latvia,

"Tartu University Clinics, Lung Hospital, Tartu,

'North Estonian Medical Centre Foundation, Centre of Pulmonology, Tallinn, Estonia,

+Otsuka Pharmaceutical Development and Commercialization, Rockville, MD, USA, and ${ }^{\S}$ Otsuka SA, Geneva, Switzerland.

CORRESPONDENCE

C.D. Wells

Otsuka Pharmaceutical Development and Commercialization 2440 Research Blvd Rockville MD 20850 USA E-mail: Charles.Wells@ otsuka-us.com

Received:

Aug 092012

Accepted after revision: Aug 242012 First published online: Sept 272012

This article has been reprinted with minor presentation changes to figure 2 .

This article was modified in April 2016 to correct errors in the licence information. 
severe form of the disease resulting in devastating consequences in some settings [13]. A recent meta-analysis of XDRTB cohorts demonstrated treatment success in only $44 \%$ of patients, with mortality in the range of $14 \%$ to $27 \%$ [14]. In the presence of HIV co-infection, mortality can exceed $70 \%$ among XDR-TB patients despite appropriate treatment for HIV [15]. Hence, better treatment options are needed to curtail the global MDR-TB and XDR-TB epidemic.

Delamanid (OPC-67683) is a new agent derived from the nitrodihydro-imidazooxazole class of compounds and inhibits mycolic acid synthesis. It has demonstrated potent pre-clinical in vitro and in vivo activity against both drug-susceptible and drugresistant strains of MTB and robust early bactericidal activity in early clinical development [16, 17]. In a randomised, placebocontrolled trial in MDR-TB patients evaluating the efficacy of 2 months of treatment of two different doses (100 mg twice daily versus $200 \mathrm{mg}$ twice daily) of delamanid in conjunction with a World Health Organization (WHO)-recommended optimised background treatment regimen (OBR) for MDR-TB, delamanid was shown to increase SCC by $\sim 50 \%$ using mycobacteria growth indicator tubes (MGIT; Becton-Dickinson, Franklin Lakes, NJ, USA) and by $>60 \%$ using solid culture media [18].

Herein, we present the treatment outcomes at 24 months for patients with pulmonary MDR- and XDR-TB who enrolled in the original randomised controlled trial, some of whom then participated in an additional 6-month open-label trial with delamanid.

\section{METHODS}

\section{Patients}

This analysis examined the effects of delamanid in combination with OBR on final treatment outcomes for MDR-TB patients who were followed through the entire course of treatment up to 24 months. Initially, all patients enrolled in a 2month treatment randomised controlled trial [18] with a subsequent subset of these patients enrolling in a 6-month, open-label treatment trial. Beyond the time of participation in either of these trials, key follow-up data on patient treatment and management through the remainder of the full treatment period was collected in an observational study. Both trials and the study were sponsored by Otsuka Pharmaceutical Development and Commercialization (Otsuka, Tokyo, Japan.) and were designed to fit within the WHO treatment paradigm for MDR-TB (fig. 1) [19]. Parent Trial 242-07-204 (Trial 204) was a double-blind, randomised controlled trial evaluating delamanid $100 \mathrm{mg}$ or $200 \mathrm{mg}$ administered twice daily for 2 months in combination with OBR in patients with pulmonary, sputum culture-positive MDR-TB (ClinicalTrials.gov identifier NCT00685360) [18]. Trial 242-07-208 (Trial 208) was a noncontrolled, open-label extension of Trial 204 that evaluated delamanid $100 \mathrm{mg}$ and/or $200 \mathrm{mg}$ twice daily in combination with OBR for an additional 6 months in those patients who completed Trial 204 (ClinicalTrials.gov identifier NCT01424670). Study 242-10-116 (Study 116) was an observational study of patients who were randomised in Trial 204 (with or without participating in Trial 208), which captured all relevant data from the microbiological assessments and clinical monitoring of these patients until the end of their treatment or until 24 months after the date of randomisation in Trial 204, whichever came first.

\section{Trial 242-07-204 and trial 242-07-208}

Trial 204 was conducted between May 2008 and June 2010 at 17 study sites in nine countries. In total, 481 patients were randomised as a part of this trial. As previously reported, participating patients were randomised to 2 months of treatment with delamanid $100 \mathrm{mg}$ twice daily plus OBR, delamanid $200 \mathrm{mg}$ twice daily plus OBR, or placebo plus OBR [18]. Treatment in a)

WHO OBR

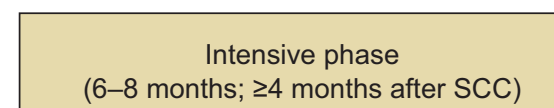

Continuation phase (12-18 months; $\geq 16$ months after SCC)

\section{Completion of the intensive phase of treatment}

Completion of the continuation phase of treatment
b)

Completion of
Trial 204
Delamanid

\begin{tabular}{|c|}
\hline Trial 204 \\
(2 months of \\
treatment)
\end{tabular}
Completion of
Completion of observation
WHO OBR
Intensive phase
(6-8 months; $\geq 4$ months after SCC)
Continuation phase (12-18 months; $\geq 16$ months after SCC)

FIGURE 1. World Health Organization (WHO) recommended treatment for multidrug-resistant tuberculosis (MDR-TB) and the design of delamanid Trial 204, Trial 208 and Study 116. a) WHO optimised background treatment regimen (OBR) recommendations for the treatment of MDR-TB [19]. b) Otsuka (Otsuka, Tokyo, Japan) design for Trial 204, Trial 208 and Study 116 (delamanid trials/study). SCC: sputum culture conversion. * : time varied between the completion of Trial 204 and the initiation of Trial 208 based on local approval processes. 
Trial 204 was administered as directly observed therapy (DOT) and patients were hospitalised for the duration of the 2-month treatment period. Safety assessments and sputum cultures were monitored weekly during treatment. Thereafter, all patients were followed on their OBR only for an additional 4 weeks with ongoing weekly assessments of safety and sputum culture status to confirm SCC.

Patients who completed participation in Trial 204 had the option to participate in Trial 208 for an additional 6 months of guaranteed access to treatment with delamanid. Trial 208 was conducted between March 2009 and October 2011 at 14 of the study sites that participated in Trial 204. In total, $213(44.2 \%)$ of the 481 patients from Trial 204 were enrolled in this trial. A gap of at least 4 weeks in delamanid treatment existed between Trial 204 and Trail 208, resulting from the Trial 204 design and the timing of local regulatory and ethics approval of Trial 208; all patients continued with OBR throughout this period. More than half $(54.5 \%)$ of the patients who participated in Trial 208 were able to resume treatment with delamanid within 2 months of completing Trial 204; however, more than one-third resumed treatment after $\geqslant 4$ months. Patients initiated treatment as a part of Trial 208 at a dose of delamanid $100 \mathrm{mg}$ twice daily, the lower dose from Trial 204. Investigators, having remained blinded to patients' Trial 204 treatment assignments, had the option to titrate up to delamanid $200 \mathrm{mg}$ twice daily after the first 2 weeks of treatment. Treatment with delamanid and OBR was administered in Trial 208 with DOT. All patients were hospitalised for 2 weeks following the initiation of treatment. Patients who had a dose titration were hospitalised for an additional 2 weeks corresponding to the initiation of treatment with the new dose. Nearly $70 \%$ of the trial participants were treated for at least 5 of the 6 months as outpatients. For all patients, OBR continued throughout their full treatment period for MDR-TB regardless of participation in Trial 208. Figure 2 presents the flow of treatment assignments for patients who participated in Trial 204 including the subset of Trial 204 patients who were then enrolled in Trial 208.

\section{Study 242-10-116: observational study}

Study 116 was a multicentre, observational study conducted between January 2011 and May 2012 at all 17 sites included in Trial 204, regardless of their participation in Trial 208 (fig. 2). The aim was to capture data for all patients that were randomised in Trial 204, therefore, the sample size was based on the number of patients participating in Trial 204 who also consented to participate in Study 116.

As previously outlined, patients continued OBR throughout their full treatment period including during participation in this study. No study-required interventions or procedures were conducted, and since no delamanid was being administered, no

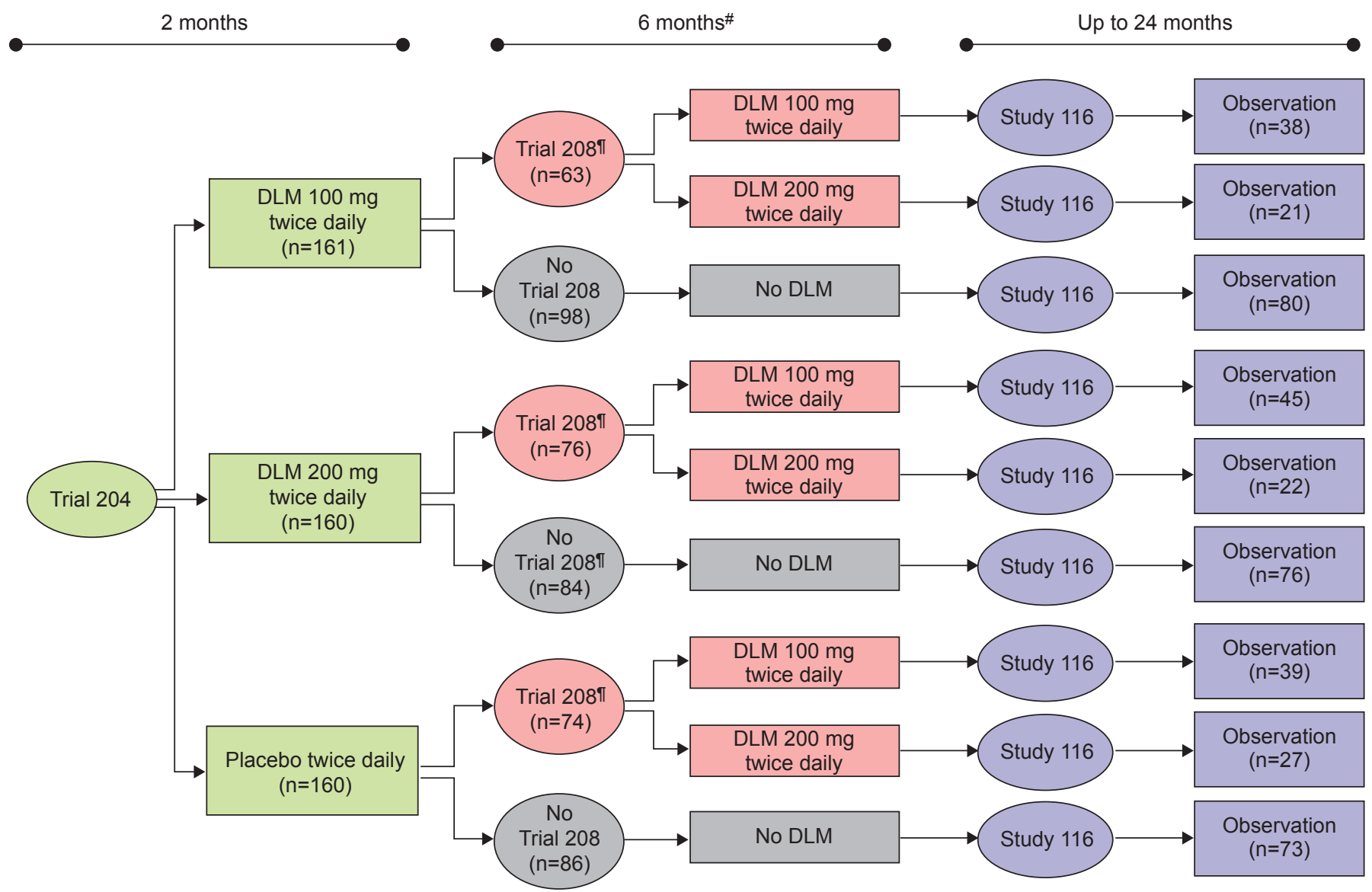

FIGURE 2. Flowchart of intent-to-treat patients in delamanid (DLM) Trial 204, Trial 208 and Study 116. *: patients who did not participate in Trial 208 were eligible for participation in Study 116 following their completion of Trial 204; " : time between the completion of Trial 204 and the initiation of Trial 208 was variable and was based on local approval processes. 
safety data was collected. Microbiological data, including results from periodic sputum culture assessments, was collected from patient medical records or from reporting systems for local TB control programmes. Patient visits occurred in the intervals specified on a local/national basis until 24 months after receiving the first dose of trial medication in Trial 204 (delamanid or placebo), or until the completion of their treatment, whichever occurred first. Data collection for Study 116 did not begin until individual participation in Trial 204 was over or between/after participation in Trial 208, if the patient also participated in the extension trial. Demographic data and the results of baseline assessments were taken from the original database from Trial 204.

\section{Optimised background treatment regimen}

The OBR employed during the two interventional trials and the observational study that provide the data for this analysis was defined on a per patient basis, according to WHO guidelines [19]. In general, OBR consisted of four to six anti-TB agents administered over the course of 18 to 24 months. The intensive phase of this regimen lasted between 6 and 8 months and usually included an injectable anti-TB agent if a patient's disease was susceptible to it. Thereafter, treatment continued with a simplified continuation phase, which generally lasted an additional 12-18 months.

\section{Analysis design}

The objective of this analysis of the combined data from Trial 204, Trial 208 and Study 116 was to assess the final treatment outcomes for patients who were initially randomised in Trial 204 and who consented to participate in Study 116. The analysis population included patients who consented to participate in Study 116, had microbiological evidence of MDR-TB prior to or at entry into Trial 204 (baseline), and had culture data using solid bacteriological media (e.g. Lowenstein-Johnson or Ogawa medium) during participation in Study 116. Although the MGIT system is more sensitive than solid media for the detection of MTB, which enhances the capacity for diagnosis and determination of SCC early in the course of treatment [18], solid media is generally used for monitoring sputum culture status during the longer course of treatment [20]. Therefore, solid media was used to determine sputum culture status. Patients who were deceased were included as a part of the analysis population. Missing culture results from the last study visit that were unknown or contaminated were imputed as a negative result only if it was preceded by two negative culture results obtained within 90 days before the date of the last visit.

Final treatment outcomes were determined by clinician's managing the patient's care. Final treatment outcomes were based on the patient's clinical status, including sputum culture status, at the end of treatment. Definitions for MDR-TB treatment outcomes were defined by WHO as follows [19]. 1) Cured: defined as a patient who completed treatment with at least five consecutive negative cultures during the last 12 months. 2) Treatment completed: defined as a patient who completed treatment but had less than five cultures performed during the last 12 months. 3) Died: defined as a patient who died for any reason while on treatment for MDR-TB. 4) Failed: defined as a patient who had two or more positive cultures among five collected during the final 12 months, or who had a positive culture among the final three sputum cultures collected from the patient as part of on-going monitoring. 5) Defaulted; defined as a patient whose treatment was interrupted for $\geqslant 2$ consecutive months for any reason without medical approval. These outcomes were subsequently grouped as either favourable (patients who met the criteria for cured or treatment completed) or unfavourable (patients who failed, died or defaulted).

In accordance with the intention to follow all patients who participated in Trial 204 and to analyse all available data, no power calculations were required. The number and percentage of patients and the corresponding $95 \%$ confidence intervals (CIs) for each outcome were summarised by the duration of treatment with any dosing regimen of delamanid. This approach was supported by available pharmacokinetic data demonstrating that patients who had been dosed with either the delamanid $100 \mathrm{mg}$ twice daily or $200 \mathrm{mg}$ twice daily dosing regimen achieved delamanid plasma exposure (area under the curve 0-24 h) in excess of the threshold range for maximal bactericidal activity $\left(3500-5500 \mathrm{~h} \cdot \mathrm{ng} \cdot \mathrm{mL}^{-1}\right.$ ) (unpublished data). This finding is further supported by the similarity of results for the delamanid $100 \mathrm{mg}$ twice daily and $200 \mathrm{mg}$ twice daily groups for the primary efficacy end-point of SCC at 2 months using the more sensitive MGIT system in Trial $204(45.4 \%$ and $41.9 \%$ SCC, respectively) [18]. Treatment groups were compared using the Cochran-Mantel-Haenszel test to determine $\mathrm{p}$-values. Point and interval estimates of risk ratios (RRs) were also calculated for treatment comparisons. Statistical analyses were conducted using SAS (version 9.2; SAS Institute, Cary, NC, USA).

\section{Ethical considerations}

Trial 204, Trial 208 and Study 116 were performed in accordance with the Good Clinical Practice guidelines of the International Conference on Harmonisation [21] and adhered to the ethical principles of the Declaration of Helsinki [22]. Trial 204, Trial 208 and Study 116 received approval by independent ethics committees/institutional review boards at all participating sites. All patients provided written informed consent in their native language before enrolment. Consent for participation in Study 116 was obtained from next of kin for patients who were deceased, in accordance with local regulations.

\section{RESULTS}

\section{Study population}

Of the 481 patients who were randomised into Trial 204 (the intent-to-treat population), all 421 (87.5\%) patients who consented to participate in Study 116 were included in the analysis population. Of these 421 patients, 225 (53.4\%) were from Asia and $278(66.0 \%)$ were male. The median age was 34 years (range 18-63 years). Only four patients were seropositive for HIV. 56 (13.3\%) patients had confirmed XDR-TB, with the remainder of patients having MDR-TB. No significant differences in demographic or baseline characteristics were identified between the 481 patients from Trial 204 and 421 patients who were included in this analysis. Full demographics and baseline clinical characteristics can be found in table 1 .

\section{Outcomes for individual treatment groups}

Of the 126 patients who received delamanid at any dose (i.e. $100 \mathrm{mg}$ twice daily and/or $200 \mathrm{mg}$ twice daily) for 8 months and the 66 patients who received delamanid at any dose for 6 months, $74.6 \%$ (94 out of 126 patients) and $74.2 \%$ (49 out of 66 patients) had favourable treatment outcomes, respectively. 


\begin{tabular}{|c|c|c|c|}
\hline TABLE 1 & \multicolumn{3}{|c|}{$\begin{array}{l}\text { Demographics and baseline clinical } \\
\text { characteristics of analysis population for } \\
\text { treatment outcomes }\end{array}$} \\
\hline \multicolumn{2}{|c|}{ Characteristics } & $\begin{array}{l}\text { Randomised in } \\
\text { Trial } 204\end{array}$ & $\begin{array}{l}\text { Analysis } \\
\text { population }^{\#}\end{array}$ \\
\hline \multicolumn{2}{|l|}{ Subjects $n$} & 481 & 421 \\
\hline \multicolumn{2}{|l|}{ Age years } & $35(18-63)$ & $34(18-63)$ \\
\hline \multicolumn{2}{|l|}{ Males } & $324(67.4)$ & $278(66.0)$ \\
\hline \multicolumn{4}{|l|}{ Region } \\
\hline \multicolumn{2}{|l|}{ Americas } & $133(27.7)$ & $125(29.7)$ \\
\hline \multicolumn{2}{|c|}{ Southeast Asia } & $150(31.2)$ & $144(34.2)$ \\
\hline \multicolumn{2}{|c|}{ Northeast Asia } & $106(22.0)$ & $81(19.2)$ \\
\hline \multicolumn{2}{|c|}{ Eastern Europe or Mediterranean } & $92(19.1)$ & $71(16.9)$ \\
\hline \multicolumn{2}{|l|}{ XDR-TB } & $72(15.0)$ & $56(13.3)$ \\
\hline \multicolumn{4}{|c|}{ Lung cavities } \\
\hline \multicolumn{2}{|l|}{ Absent } & $153(31.8)$ & $137(32.5)$ \\
\hline \multicolumn{2}{|l|}{ Unilateral } & $213(44.3)$ & $188(44.7)$ \\
\hline \multicolumn{2}{|l|}{ Bilateral } & $115(23.9)$ & $96(22.8)$ \\
\hline \multicolumn{4}{|c|}{ Previous treatment } \\
\hline \multicolumn{2}{|c|}{ First-line only } & $295(61.3)$ & $272(64.6)$ \\
\hline \multicolumn{2}{|c|}{$\begin{array}{l}\text { Second-line with or without } \\
\text { first-line }\end{array}$} & $138(28.7)$ & $110(26.1)$ \\
\hline \multicolumn{2}{|c|}{$\begin{array}{l}\text { Third-line with or without first-line } \\
\text { or second-line }\end{array}$} & $48(10)$ & $39(9.3)$ \\
\hline
\end{tabular}

Data are presented as median (range) or $\mathrm{n}(\%)$, unless otherwise stated. XDRTB: extensively drug-resistant tuberculosis. ${ }^{\#}$ : included all patients randomised in Trial 204 who also consented to participate in Study 116, and who had microbiological data to support assessment of treatment outcomes using solid culture media.

Because of the similarity of favourable outcomes following treatment with delamanid at any dose for 8 or 6 months, the patients from these two treatment groups were combined into a single long-term treatment group $(n=192)$ for subsequent analyses. The remaining 229 patients in the analysis population received up to 2 months of treatment with delamanid $(n=156)$ at either dose (100 mg twice daily or $200 \mathrm{mg}$ twice daily) or placebo $(n=73)$ as a part of Trial 204. Treatment with delamanid was previously shown in Trial 204 to improve SCC after 2 months [18]. However, as with rifampicin-based TB treatment regimens [23], outcomes for patients who received a limited treatment course of only 2 months of delamanid during the full period of MDR-TB treatment did not differ from those of patients treated with OBR and placebo. Favourable outcomes were observed in $53.8 \%$ (84 out of 156 patients) who received delamanid at either dose for only 2 months and in 57.5\% (42 out of 73 patients) who received no delamanid. Therefore, since the combination of OBR with delamanid for only 2 months yielded similar long-term treatment outcomes to the combination of OBR with placebo, patients from these two treatment groups were combined into a single short-term treatment group $(n=229)$ for subsequent analyses.

\section{Overall outcomes}

Overall, favourable outcomes were significantly increased in patients in the long-term ( $\geqslant 6$ months) treatment group at
$74.5 \%$ (95\% CI $67.7-80.5 \%$ ), as compared to $55.0 \%$ (95\% CI $48.3-61.6 \%$ ) among those in the short-term ( $\leqslant 2$ months) treatment group who were treated with delamanid or placebo for 2 months (RR 1.35, 95\% CI 1.17-1.56; p<0.001) (table 2). Of note, $17.2 \%$ of patients completed treatment in the long-term delamanid group, as compared with $6.6 \%$ of patients who completed treatment in the short-term delamanid group (RR 2.624, $95 \%$ CI 1.47-4.68; p<0.001). To qualify as "treatment completers", patients must have completed treatment according to the treatment programme protocols [19]. These patients did not meet the definition of cure because of a more limited amount of bacteriological results available (i.e. fewer than five cultures were performed in the final 12 months of treatment). This is not unexpected as it may reflect patients who have had substantial clinical improvement earlier in the course of treatment and who may be less able to produce sputum for culture assessment late in the course of treatment. Patient mortality was also greatly reduced following extended treatment with delamanid. Only two $(1.0 \%)$ deaths occurred in the long-term treatment group, while 19 (8.3\%) occurred in the short-term treatment group; this difference was statistically significant (RR $0.13,95 \%$ CI $0.03-$ $0.53 ; \mathrm{p}<0.001)$.

\section{XDR-TB outcomes}

A higher proportion of favourable outcomes were also observed in the subset of XDR-TB patients after extended treatment with delamanid. Among these patients, 61.4\% (95\% CI 45.5-75.6\%) experienced favourable outcomes (table 3), while only $50.0 \%$ (95\% CI $21.1-78.9 \%)$ of patients in the short-term treatment group had favourable outcomes (table 3). As with MDR-TB more broadly, mortality for the subset of XDR-TB patients was reduced among those who received extended treatment with delamanid. No deaths occurred among these XDR-TB patients while three $(25.0 \%)$ deaths occurred in the short-term treatment group; this difference was statistically significant $(\mathrm{p}<0.001)$.

\section{DISCUSSION}

This is the first reported analysis of treatment outcomes for MDR- and XDR-TB patients who were treated with delamanid, a new and novel anti-TB medication. This analysis demonstrates that a higher proportion of patients achieve favourable outcomes and have lower mortality when treated with delamanid for at least 6 months in combination with a WHO-recommended OBR. These results build on the previously reported positive findings that adding delamanid to OBR increases SCC among patients earlier in the course of MDR-TB treatment [18], and are compelling when compared to published reports of MDR-TB treatment outcomes [5-7]. Even in selected cohorts of MDR-TB patients, with disease known to be susceptible to a fluoroquinolone and three or more injectable products, the proportion of patients experiencing favourable outcomes rarely exceeds $70 \%$ and mortality usually exceeds $15 \%$ [24]. Under programmatic conditions in high resource settings such as the EU, published studies report favourable outcomes ranging from $18.2 \%$ to $68.5 \%$ [12]. Similarly, reported mortality among MDRTB cohorts far exceeds the $1.0 \%$ measured in this analysis [5-7] and has been shown to be higher among patients who fail to achieve SCC early in the course of treatment [9]. Likewise, in the subgroup of XDR-TB patients, a greater proportion achieved favourable outcomes and had lower mortality following 


\begin{tabular}{|c|c|c|c|}
\hline Treatment outcome & Long-term treatment ${ }^{\#}$ & Short-term treatment & All patients ${ }^{+}$ \\
\hline Favourable & $143(74.5 ; 67.7-80.5)^{\S}$ & $126(55.0 ; 48.3-61.6)^{5}$ & 269 (63.9; 59.1-68.5) \\
\hline Cured & $110(57.3 ; 50.0-64.4)$ & $111(48.5 ; 41.8-55.1)$ & $221(52.5 ; 47.6-57.4)$ \\
\hline Completed & $33(17.2 ; 12.1-23.3)^{5}$ & $15(6.6 ; 3.7-10.6)^{5}$ & $48(11.4 ; 8.5-14.8)$ \\
\hline Died & $2(1.0 ; 0.1-3.7)^{\S}$ & $19(8.3 ; 5.1-12.7)^{5}$ & $21(5.0 ; 3.1-7.5)$ \\
\hline Failed & $32(16.7 ; 11.7-22.7)$ & $26(11.4 ; 7.6-16.2)$ & $58(13.8 ; 10.6-17.4)$ \\
\hline Defaulted & $15(7.8 ; 4.4-12.6)^{5}$ & $58(25.3 ; 19.8-31.5)^{\S}$ & 73 (17.3; 13.8-21.3) \\
\hline
\end{tabular}

Data are presented as n (\%; 95\% CI). MDR: multidrug-resistant; TB: tuberculosis; XDR: extensively drug-resistant. \#: 192 patients received delamanid (100 mg and/or $200 \mathrm{mg}$ twice a day) for at least 6 months; ${ }^{*}: 229$ patients received delamanid (100 mg or $200 \mathrm{mg}$ twice a day) or placebo for 2 months; ${ }^{+}: \mathrm{n}=421 ;{ }^{\S}:$ differences between the long-term and the short-term treatment groups for the corresponding treatment outcome were statistically significant $(p<0.001)$, all other differences did not reach statistical significance $(p \geqslant 0.05)$.

long-term treatment with delamanid ( $\geqslant 6$ months). Notably, all 44 XDR-TB patients who had received delamanid for at least 6 months survived.

Therefore, our results have important implications for MDRand XDR-TB patients given the considerable improvements in morbidity and mortality that have been documented following treatment with delamanid for 6 months when added to an OBR. These results, combined with the improvements in earlier SCC previously documented from treatment with delamanid [18], also have important implications for TB control by potentially leading to a reduction in transmission of MDR- and XDR-TB from patients who would be treated more effectively.

It is important to note that although patients were hospitalised throughout the Trial 204 treatment period, Trial 208 was conducted primarily as an outpatient trial following a brief initial period of required hospitalisation. Both Trial 204 and Trial 208 were conducted with patients being administered treatment as DOT. Trial 208, conducted largely as an outpatient trial, reflected more typical programmatic conditions in its delivery of treatment and care for MDR-TB.

As the inappropriate use of anti-TB drugs continues to be a major problem worldwide $[25,26]$, the efficacy of delamanid, as demonstrated in the clinical trial setting, will need to be preserved by its rational use. Recent studies have demonstrated that even among European MDR-TB reference centres, misuse of TB drugs occurs in up to $20 \%$ of cases [27]. Furthermore, a recent meta-analysis demonstrated a strong correlation between the misuse of anti-TB drugs and drug resistance [28]. This further emphasises the need for assuring the rational use of novel TB and MDR-TB drugs [29, 30].

Several limitations exist with the interpretations of our results. First, although these results for XDR-TB patients are encouraging, they should be interpreted cautiously in light of the relatively small sample size. Secondly, patient follow-up was carried out under programmatic conditions where participating organisations and treatment programmes had variable processes and procedures, including timing of sputum culture assessments. This created challenges in Study 116 for the collection and alignment of available microbiological data for analysis and determination of bacteriological outcomes (i.e. cure versus treatment completion) as defined by WHO. Thirdly, as Study 116 was initiated close to the completion of Trial 204, with retrospective capture of data for some patients completing Trial 204 earlier in its conduct, information bias may have been introduced. Fourthly, a considerable gap in delamanid

TABLE 3 Long-term (24 month) treatment outcomes after treatment with delamanid in combination with an optimised background treatment regimen: XDR-TB patients only

\begin{tabular}{lccc} 
Treatment outcome & Long-term treatment ${ }^{\#}$ & Short-term treatment & All Patients $^{+}$ \\
\hline Favourable & $27(61.4 ; 45.5-75.6)$ & $6(50.0 ; 21.1-78.9)$ & $33(58.9 ; 45.0-71.9)$ \\
Cured & $11(25.0 ; 13.2-40.3)$ & $5(41.7 ; 15.2-72.3)$ & $16(28.6 ; 17.3-42.2)$ \\
Completed & $16(36.4 ; 22.4-52.2)$ & $1(8.3 ; 0.2-38.5)$ & $17(30.4 ; 18.8-44.1)$ \\
Unfavourable & $17(38.6 ; 24.4-54.5)$ & $6(50.0 ; 21.1-78.9)$ & $23(41.1 ; 28.1-55.0)$ \\
Died & $0(0.0)^{\mathbf{S}}$ & $3(25.0 ; 5.5-57.2)^{\mathbf{S}}$ & $3(5.4 ; 1.1-14.9)$ \\
Failed & $14(31.8 ; 18.6-47.6)$ & $3(25.0 ; 5.5-57.2)$ & $17(30.4 ; 18.8-44.1)$ \\
Defaulted & $3(6.8 ; 1.4-18.7)$ & $0.0(0.0)$ & $3(5.4 ; 1.1-14.9)$ \\
\hline
\end{tabular}

Data are presented as n (\%; 95\% Cl). XDR-TB: extensively drug-resistant tuberculosis. \# ${ }^{*} 44$ patients received delamanid (100 mg and/or $200 \mathrm{mg}$ twice a day) for at least 6 months; ${ }^{*}: 12$ patients received delamanid (100 mg or $200 \mathrm{mg}$ twice a day) or placebo for 2 months; ${ }^{+}: n=56$; ${ }^{5}$ : differences between the long-term and the short-term treatment groups for the corresponding treatment outcome were statistically significant $(p<0.001)$, all other differences did not reach statistical significance $(p \geqslant 0.05)$. 
treatment between parent Trial 204 and extension Trial 208 occurred for some patients. Although more than half of the patients who went on to participate in Trial 208 resumed treatment with delamanid within 2 months, approximately onethird did so $>4$ months later due to delayed local trial approval processes. Finally, because clinicians remained blinded to Trial 204 treatment assignments throughout the conduct of Trial 208 and had the option to titrate their patients up to delamanid $200 \mathrm{mg}$ twice daily from the initial dose of delamanid $100 \mathrm{mg}$ twice daily, patients who received delamanid for a total of 8 months as a part of participation in both trials may have experienced variability in the delamanid dose administered across the two trials. However, this is mitigated by evidence suggesting that dosing with either delamanid $100 \mathrm{mg}$ twice daily or $200 \mathrm{mg}$ twice daily results in concentrations above the exposure threshold for maximal bactericidal activity (unpublished data).

\section{Conclusions}

This analysis of 24 months of follow-up of MDR- and XDR-TB patients who were treated with delamanid in combination with a WHO-recommended OBR, demonstrated substantially improved treatment outcomes for those patients who received $\geqslant 6$ months of delamanid, including a statistically significant reduction in mortality. Taken together with the positive results demonstrated in the previously reported randomised controlled trial, delamanid demonstrates great promise as a new therapy for the treatment of MDR-TB when used in conjunction with OBR. Another large randomised controlled trial examining 6 months of treatment with delamanid in combination with a background treatment regimen is enrolling patients with MDR$\mathrm{TB}$, and will include patients who have HIV co-infection and who are receiving anti-retroviral drugs (ClinicalTrials.gov identifier NCT01424670). While awaiting results from ongoing phase III trials and further clinical development of new anti-TB drugs, greater priority should be placed on ensuring rational use of anti-TB drugs more broadly, as doing so will contribute to the longevity of new and promising compounds such as delamanid.

\section{STATEMENT OF INTEREST}

Conflict of interest information can be found alongside the online version of this article at www.erj.ersjournals.com

\section{ACKNOWLEDGEMENTS}

We thank the many primary investigators and co-investigators for their expert clinical management of patients and guidance during the conduct of Trial 204, Trial 208 and Study 116.

\section{REFERENCES}

1 World Health Organization. Towards Universal Access to Diagnosis and Treatment of Multidrug-Resistant and Extensively Drug-Resistant Tuberculosis by 2015: WHO Progress Report 2011. Geneva, World Health Organization, 2011.

2 Falzon D, Jaramillo E, Schünemann HJ, et al. WHO guidelines for the programmatic management of drug-resistant tuberculosis: 2011 update. Eur Respir J 2011; 38: 516-528.

3 Diel R, Rutz S, Castell S, et al. Tuberculosis: cost of illness in Germany. Eur Respir J 2012; 40: 143-151.

4 Loddenkemper R, Sotgiu G, Mitnick CD. Cost of tuberculosis in the era of multidrug resistance: will it become unaffordable? Eur Respir J 2012; 40: 9-11.
5 Orenstein EW, Basu S, Shah NS, et al. Treatment outcomes among patients with multidrug-resistant tuberculosis: systematic review and meta-analysis. Lancet Infect Dis 2009; 9: 153-161.

6 Johnston JC, Shahidi NC, Sadatsafavi M, et al. Treatment outcomes of multidrug-resistant tuberculosis: a systematic review and metaanalysis. PLoS One 2009; 4: e6914.

7 Ahuja SD, Ashkin D, Avendano M, et al. Multidrug resistant pulmonary tuberculosis treatment regimens and patient outcomes: an individual patient data meta-analysis of 9,153 patients. PLoS Med 2012; 9: e1001300.

8 Holtz TH, Sternberg M, Kammerer S, et al. Time to sputum culture conversion in multidrug-resistant tuberculosis: predictors and relationship to treatment outcome. Ann Intern Med 2006; 144: 650-659.

9 Kurbatova EV, Taylor A, Gammino VM, et al. Predictors of poor outcomes among patients treated for multidrug-resistant tuberculosis at DOTS-plus projects. Tuberculosis (Edinb) 2012; 92: 397-403.

10 Manissero D, Hollo V, Huitric E, et al. Analysis of tuberculosis treatment outcomes in the European Union and European Economic Area: efforts needed towards optimal case management and control. Euro Surveill 2010; 15: pii 19514.

11 European Centre for Disease Prevention and Control/WHO Regional Office for Europe: Tuberculosis surveillance in Europe 2008. Stockholm, European Centre for Disease Prevention and Control, 2010.

12 van Hest R, Ködmön C, Verver S, et al. Tuberculosis treatment outcome monitoring in European Union countries: systematic review. Eur Respir J 2013; 41: 635-643.

13 Sotgiu G, Ferrara G, Matteelli A, et al. Epidemiology and clinical management of XDR-TB: a systematic review by TBNET. Eur Respir J 2009; 33: 871-881.

14 Jacobson KR, Tierney DB, Jeon CY, et al. Treatment outcomes among patients with extensively drug-resistant tuberculosis: systematic review and meta-analysis. Clin Infect Dis 2010; 51: 6-14.

15 Gandhi NR, Moll A, Sturm AW, et al. Extensively drug-resistant tuberculosis as a cause of death in patients co-infected with tuberculosis and HIV in a rural area of South Africa. Lancet 2006; 368: 1575-1580.

16 Matsumoto M, Hashizume $\mathrm{H}$, Tomishige $\mathrm{T}$, et al. OPC-67683, a nitro-dihydro-imidazooxazole derivative with promising action against tuberculosis in vitro and in mice. PLoS Med 2006; 3: e466.

17 Diacon $\mathrm{AH}$, Dawson R, Hanekom M, et al. Early bactericidal activity of delamanid (OPC-67683) in smear-positive pulmonary tuberculosis patients. Int J Tuberc Lung Dis 2011; 15: 949-954.

18 Gler MT, Skripconoka V, Sanchez-Garavito E, et al. Delamanid for multidrug-resistant pulmonary tuberculosis. N Engl J Med 2012; 366: 2151-2160.

19 World Health Organization. Guidelines for the Programmatic Management of Drug-Resistant Tuberculosis. Geneva, World Health Organization, 2008.

20 Gammino VM, Taylor AB, Rich ML, et al. Bacteriologic monitoring of multidrug-resistant tuberculosis patients in five DOTS-Plus pilot projects. Int J Tuberc Lung Dis 2011; 15: 1315-1322.

21 International Conference on Harmonisation of Technical Requirements of Registration for Pharmaceuticals for Human Use. ICH Harmonised Tripartite Guideline: Guideline for Good Clinical Practice, E6 (R1). 1996.

22 World Medical Association Declaration of Helsinki - Ethical Principles for Medical Research Involving Human Subjects. www. wma.net/en/30publications/10policies/b3/ Date last accessed: August 7, 2012

23 Fox W, Ellard GA, Mitchison DA. Studies on the treatment of tuberculosis undertaken by the British Medical Research Council tuberculosis units, 1946-1986, with relevant subsequent publications. Int J Tuberc Lung Dis 1999; 3: Suppl. 2, S231-S279. 
24 Migliori GB, Lange C, Centis R, et al. Resistance to second-line injectables and treatment outcomes in multidrug-resistant and extensively drug-resistant tuberculosis cases. Eur Respir J 2008; 31 : 1155-1159.

25 Zumla A, Blasi F, Raviglione M. Rational use of anti-tuberculosis drugs in the EU: better patient care and less drug resistance. Eur Respir J 2012; 39: 802-804.

26 Raviglione MC, Lange C, Migliori GB. Preventing and managing antimicrobial resistance: imperative for chest physicians. Eur Respir J 2011; 37: 978-981.
27 Migliori GB, Sotgiu G, D'Ambrosio L, et al. TB and MDR/XDR-TB in European Union and European Economic Area countries: managed or mismanaged? Eur Respir J 2012; 39: 619-625.

28 van der Werf MJ, Langendam MW, Huitric E, et al. Multidrug resistance after inappropriate tuberculosis treatment: a metaanalysis. Eur Respir J 2012; 39: 1511-1519.

29 Migliori GB, Sotgiu G. Treatment of tuberculosis: have we turned the corner? Lancet 2012; 380: 955-957.

30 D'Ambrosio L, Centis R, Migliori GB. Multidrug-resistant tuberculosis. N Engl J Med 2012; 367: 2154; author reply 2155-6. 